Pacific

Journal of

Mathematics

\title{
GROUPS WITH TORSION, BORDISM AND RHO INVARIANTS
}

Paolo Piazza And Thomas Schick 


\title{
GROUPS WITH TORSION, BORDISM AND RHO INVARIANTS
}

\author{
PAOlO PIAZZA AND ThOMAS SCHICK
}

Let $\Gamma$ be a discrete group, and let $M$ be a closed spin manifold of dimension $m>3$ with $\pi_{1}(M)=\Gamma$. We assume that $M$ admits a Riemannian metric of positive scalar curvature. We discuss how to use the $L^{2}$-rho invariant $\rho_{(2)}$ and the delocalized eta invariant $\eta_{<g>}$ associated to the Dirac operator on $M$ to get information about the space of metrics with positive scalar curvature.

In particular, we prove that if $\Gamma$ contains torsion and $m \equiv 3(\bmod 4)$ then $M$ admits infinitely many different bordism classes of metrics with positive scalar curvature. This implies that there exist infinitely many concordance classes; we show that this is true even up to diffeomorphism.

If $\Gamma$ has certain special properties, for example, if it contains polynomially growing conjugacy classes of finite order elements, then we obtain more refined information about the "size" of the space of metrics of positive scalar curvature, and these results also apply if the dimension is congruent to $1 \bmod 4$. For example, if $\operatorname{dim} M \equiv 1(\bmod 4)$ and $\Gamma$ contains a central element of odd order, then the moduli space of metrics of positive scalar curvature (modulo the action of the diffeomorphism group) has infinitely many components, if it is not empty.

Some of our invariants are the delocalized eta invariants introduced by John Lott. These invariants are defined by certain integrals whose convergence is not clear in general, and we show, in effect, that examples exist where this integral definitely does not converge, thus answering a question of Lott.

We also discuss the possible values of the rho invariants of the Dirac operator and show that there are certain global restrictions (provided that the scalar curvature is positive).

\section{Introduction and main results}

Let $M$ be a closed smooth manifold with fundamental group $\Gamma$ and universal cover $\bar{M}$. In this paper, we are concerned mainly with the set $\mathbb{R}^{+}(M)$ of metrics of positive scalar curvature on $M$ (this is in fact a topological space).

MSC2000: $58 \mathrm{~J} 28$.

Keywords: spin manifolds, positive scalar curvature, Dirac operators, rho invariants, bordism. 
There is of course a preliminary question, namely whether this space is nonempty. It is known that there are powerful obstructions to the existence of positive scalar curvature ( $\equiv$ PSC) metrics, the most successful being the one implied by the Lichnerowicz formula: on a spin manifold with positive scalar curvature, the spin Dirac operator twisted by the Mishchenko line bundle $\mathscr{V}:=\bar{M} \times_{\Gamma} C_{r}^{*} \Gamma$ is invertible. In this paper we shall leave the existence problem aside and assume that there exists a metric with positive scalar curvature. We shall instead concentrate on the classification question: if one such metric exists, how many can we put on $M$ that are distinct? We need to clarify what we mean by distinct. There are three ways for distinguishing two metrics of positive scalar curvature $g_{1}$ and $g_{2}$ on $M$.

The first one is to say that $g_{1}$ and $g_{2}$ are not path-connected in $\mathbb{R}^{+}(M)$. Thus, in this case, we are interested in $\pi_{0}\left(\mathscr{R}^{+}(M)\right)$, the set of arcwise connected components of $\mathscr{R}^{+}(M)$.

The second way for distinguishing two PSC metrics employs the notion of concordance: $g_{1}$ and $g_{2}$ are concordant if there exists a metric of PSC on $M \times[0,1]$ extending $g_{1}$ on $M \times\{0\}, g_{2}$ on $M \times\{1\}$ and of product-type near the boundary. The set of concordance classes of PSC metrics on $M$ is denoted by $\tilde{\pi}_{0}\left(\mathbb{R}^{+}(M)\right)$.

Convention 1.1. Throughout the paper, whenever we work with a Riemannian metric on a manifold with boundary, we assume that the metric has product structure near the boundary. Observe, in particular, that the restriction to the boundary of such a metric has positive scalar curvature if the original one has positive scalar curvature.

The third and more subtle way for distinguishing two PSC metrics $g_{1}, g_{2}$ on a spin manifold $M$ employs the notion of bordism.

Definition 1.2. Let $M$ be a closed spin manifold with fundamental group $\Gamma$. Two metrics $g_{1}$ and $g_{2}$ of positive scalar curvature on $M$ are $\pi_{1}$-spin bordant if there is a compact spin manifold $W$ with positive scalar curvature metric $g$ and with boundary $\partial W=\left(M, g_{1}\right) \amalg\left(-M, g_{2}\right)$, which admits a $\Gamma$-covering $\bar{W}$ whose boundary is the union of the universal coverings of the two boundary components.

Note that this notion has an evident extension to metrics on possibly different spin manifolds.

It is obvious that if two metrics $\left(g_{1}, g_{2}\right)$ are concordant, then they are, in particular, bordant, since we can choose $W=[0,1] \times M$ as the underlying manifold of the bordism. (On the other hand there are examples of nonconcordant metrics that are bordant, see [Lawson and Michelsohn 1989, page 329].) It is also rather clear that two metrics that lie in the same path component of the space of all metrics of positive scalar curvature on a given manifold $M$ are concordant and, therefore, bordant. Summarizing, as far as the problem of distinguishing metrics of positive 
scalar curvature is concerned, we have:

$$
\text { nonbordant } \Rightarrow \text { nonconcordant } \Rightarrow \text { non-path-connected. }
$$

In this paper we shall use the $L^{2}$-rho invariant $\rho_{(2)}$ of Cheeger-Gromov and the delocalized eta invariant $\eta_{<g>}$ of Lott for the spin Dirac operator associated to $(M, g)$ to distinguish nonbordant metrics of positive scalar curvature. Fundamental to our analysis will be the bordism invariance of $\rho_{(2)}$ and $\eta_{<g>}$, the long exact sequence of bordism groups due to Stephan Stolz, and some fundamental examples due to Botvinnik and Gilkey.

To apply our methods, pioneered by Botvinnik and Gilkey [1995], we shall need to assume that $\Gamma \equiv \pi_{1}(M)$ is not torsion-free: indeed if $\Gamma$ is torsion free and satisfies the Baum-Connes conjecture for the maximal group $C^{*}$-algebra then, because of the PSC assumption, these invariants are identically zero, as we have proved in [Piazza and Schick 2007] ${ }^{1}$; moreover, there are no known examples of torsion-free groups for which these invariants are nonzero.

As an example of the results we shall establish, we anticipate one of our main theorems:

Theorem 1.3. Assume that $M$ is a spin manifold of dimension $4 k+3$, where $k>0$. Assume that $g$ is a metric with positive scalar curvature on $M$ and that the fundamental group $\Gamma$ of $M$ contains torsion. Then $M$ admits infinitely many different $\Gamma$-bordism classes of metrics with $\mathrm{scal}>0$; they are distinguished by $\rho_{(2)}$. These infinitely many bordism classes remain distinct even after we mod out the action of the group of diffeomorphisms of $M^{2}$.

This theorem generalizes results of [Botvinnik and Gilkey, 1995; 1996]; other generalizations of their results include [Leichtnam and Piazza 2001].

Under additional assumptions on the group $\Gamma$ we shall be able to estimate the size of the set of equivalence classes of nonbordant metrics by proving that a free group of a certain rank acts freely on this set. We want to single out one consequence of these results, which also apply in dimensions $4 k+1$ :

Corollary 1.4. If $\Gamma$ is a discrete group which contains a central element of odd order, and if $M$ is a spin manifold with fundamental group $\Gamma$ and of dimension $4 k+1$ that admits a metric with positive scalar curvature, then the moduli space of such metrics (modulo the action of the diffeomorphism group via pullback) has infinitely many components:

$$
\left|\pi_{0}\left(\mathscr{R}^{+}(M) / \operatorname{Diffeo}(M)\right)\right|=\infty .
$$

\footnotetext{
${ }^{1}$ For the delocalized eta invariant, it suffices to assume that $\Gamma$ satisfies the Baum-Connes conjecture for the reduced $C^{*}$-algebra.

${ }^{2}$ For the precise meaning of this, compare Theorem 2.25.
} 
To our knowledge, this is the first general result of this kind that applies in dimensions congruent to 1 modulo 4 .

By combining the methods of this paper with those of [Leichtnam and Piazza 2001], it should be possible to extend to even-dimensional manifolds with special fundamental groups.

Note that if the dimension is congruent to $3 \bmod 4$, then it is always true that (1.5) holds; compare [Lawson and Michelsohn 1989, Theorem 7.7 of Chapter IV].

There is a parallel story for the signature operator, where the condition on positive scalar curvature is replaced by "homotopy invariance" - stated differently, one gets vanishing or classification results for the disjoint union of one manifold with a homotopy equivalent second manifold.

For instance, if $\Gamma$ is torsion-free and satisfies the Baum-Connes conjecture for the maximal $C^{*}$-algebra, we prove in [Piazza and Schick 2007] that $\rho_{(2)}$ and $\eta_{<g>}$ vanish on a manifold that is the disjoint union of two homotopy equivalent manifolds. For $\eta_{(2)}$, this result is originally due to Keswani [2000].

Similarly, the nontriviality result we give in Theorem 1.3 has a relative for the signature operator, which actually motivated our result and its proof:

Theorem 1.6 [Chang and Weinberger 2003]. If $M$ is a compact, oriented manifold of dimension $4 k+3$ with $k>0$ such that $\pi_{1}(M)$ is not torsion-free, then there are infinitely many manifolds that are homotopy equivalent to $M$ but not homeomorphic to it.

Note in particular that the structure set $\mathscr{S}(M)$ has infinite cardinality. Chang and Weinberger ask about more precise results concerning the "size" of the structure set if the fundamental group contains a lot of torsion. In this paper, we investigate the corresponding question for the space of metrics of positive scalar curvature and use in particular the delocalized eta invariants of John Lott to get some positive results - for precise statements, consult Theorem 2.27 and Theorem 2.32. It should be possible, although technically more difficult given that the boundary operator is not invertible, to extend the results stated in those two theorems to the signature operator and the structure set of a fixed manifold. We plan to investigate this and further directions of research in future work.

Our results rely on the delocalized eta invariants of Lott [1999], applied in those situations where they are well defined and one does not have any convergence problems (for example, for central group elements). However, we give examples in Section 3 that show that in general the convergence one hopes for does definitely not occur, showing the limitations of this method.

Closely related to the delocalized rho invariants we consider are those associated to virtual representations of dimension zero (we explain the translation between 
the two points of view via "Fourier transform" in Section 2A). Given such a finitedimensional virtual unitary representation $\left[\lambda_{1}-\lambda_{2}\right]$, let $F_{1}$ and $F_{2}$ be the associated flat vector bundles. Then the corresponding rho invariant is simply

$$
\rho_{\lambda_{1}-\lambda_{2}}(\not D)=\eta\left(\not D_{F_{1}}\right)-\eta\left(\not D_{F_{2}}\right) .
$$

One might wonder what the possible values of the rho invariants are, if the group is not torsion-free. For the signature operator and these APS-rho invariants, a result of this type has been proved by Guentner-Higson-Weinberger:

Theorem 1.7 [Guentner et al. 2005, Theorem 7.1]. Let $M$ and $N$ be smooth, closed, oriented, odd-dimensional manifolds which are oriented homotopy equivalent. Let $\Gamma:=\pi_{1}(M)$ and let $\lambda_{1}, \lambda_{2}: \Gamma \rightarrow U(d)$ be two unitary representations. Let $R^{\prime}$ be the smallest subring of $\mathbb{Q}$ generated by $\mathbb{Z}, 1 / 2$, and $o\left(\lambda_{1}(g)\right)^{-1}$ and $o\left(\lambda_{2}(g)\right)^{-1}$ for each $g \in \Gamma$. Here, $o(x)$ is the order of the group element $x \in U(d)$, and we set $(+\infty)^{-1}:=0$. Then

$$
\rho_{\lambda_{1}-\lambda_{2}}\left(D_{M}^{\text {sign }}\right)-\rho_{\lambda_{1}-\lambda_{2}}\left(D_{N}^{\text {sign }}\right) \in R^{\prime} .
$$

We end the paper by proving the corresponding result in the positive scalar curvature context. It is no surprise that we don't need to invert 2 , as is notoriously necessary in L-theory contexts.

Theorem 1.8. Let $M$ be a spin manifold with a Riemannian metric of positive scalar curvature and let $\not D$ be the associated Dirac operator. Let $\lambda_{j}$ be as in Theorem 1.7 and let $R$ be the smallest subring of $\mathbb{Q}$ generated by $\mathbb{Z}$ and $o\left(\lambda_{1}(G)^{-1}\right.$, $o\left(\lambda_{2}(g)^{-1}\right.$ for each $g \in \Gamma$ of finite order. Then

$$
\rho_{\lambda_{1}-\lambda_{2}}(\not D) \in R
$$

\section{Distinguishing metrics with positive scalar curvature}

2A. Torsion in $\pi_{1}(M)$ and $\operatorname{dim} M \equiv 3(\bmod 4)$. We will frequently talk about spin manifolds; we think of them as being manifolds with a given spin structure (so they correspond to oriented manifolds, not to orientable manifolds).

Let $X$ be any space. Then there is an exact sequence of bordism groups due to Stephan Stolz, see [Stolz 1995, p. 630], [Stolz 1999].

$$
\rightarrow \Omega_{n+1}^{\mathrm{spin}}(X) \stackrel{t}{\rightarrow} R_{n+1}^{\mathrm{spin}}(X) \stackrel{\delta}{\rightarrow} \operatorname{Pos}_{n}^{\mathrm{spin}}(X) \rightarrow \Omega_{n}^{\mathrm{spin}}(X) \rightarrow R_{n}^{\mathrm{spin}}\left(\pi_{1}(X)\right) \rightarrow
$$

Recall the definition of the terms in the sequence:

Definition 2.2. (a) First, $\Omega_{*}^{\text {spin }}(X)$ is the singular spin bordism group of $X$, the set of closed spin manifolds with a reference map to $X$, modulo spin bordism. 
(b) $\operatorname{Pos}_{*}^{\text {spin }}(X)$ is the bordism group of spin manifolds with a given metric with scal $>0$, with a reference map to $X$. A bordism in $\operatorname{Pos}_{*}^{\text {spin }}(X)$ is a bordism of spin manifolds as above together with a metric with positive scalar curvature that restricts to the given metrics at the boundary (with a product structure near the boundary).

(c) $R_{*}^{\text {spin }}(X)$ is the set of compact spin manifolds with boundary, where the boundary is equipped with a metric having positive scalar curvature together with a reference map to $X$, modulo bordism. A bordism consists first of a bordism of the boundary with a metric having scal $>0$, as in the bordism relation for $\operatorname{Pos}_{*}^{\text {spin }}$. Glue this bordism of the boundary to the original manifold with boundary to get a closed manifold. The second part of a bordism in $R_{*}^{\text {spin }}(X)$ is a zero bordism of the resulting closed manifold (together with an extension of the reference map).

(d) The maps in the exact sequence (2.1) are quite obvious: $R_{n+1}^{\text {spin }} \rightarrow \operatorname{Pos}_{n}^{\text {spin }}$ is given by taking the boundary, $\operatorname{Pos}_{n}^{\text {spin }} \rightarrow \Omega_{n}^{\text {spin }}$ by forgetting the Riemannian metric, and $\Omega_{n}^{\text {spin }} \rightarrow R_{n}^{\text {spin }}$ by understanding a closed manifold as a manifold with empty boundary, this boundary therefore having a metric with scal $>0$.

(e) The sequence is exact by definition. It is also evident that this sequence is natural with respect to maps $X \rightarrow Y$, and each entry is a covariant functor with respect to such maps.

Definition 2.3. Let $M$ be a closed spin manifold with fundamental group $\Gamma$. Let $u: M \rightarrow B \Gamma$ be a classifying map for a universal covering (that is, an isomorphism on $\left.\pi_{1}\right)$. We set charPos ${ }^{\text {spin }}(M, u):=\left\{[M, g, u] \in \operatorname{Pos}_{*}^{\text {spin }}(B \Gamma)\right\}$. These are all the different bordism classes of metrics with positive scalar curvature on $M$ (where bordisms are considered that respect the given map $u$, that is, they include the data of the fundamental group, and where also the spin structure on $M$ is fixed once and for all). Note that charPos $\operatorname{Ppin}^{\operatorname{spin}}(M, u)$ is a subset of the group $\operatorname{Pos}_{n}^{\text {spin }}(B \Gamma)$, but we can't expect that it is a subgroup.

In this section, we will study the set $\operatorname{charPos}^{\operatorname{spin}}(M, u)$; we denote the class $[M, g, u] \in \operatorname{charPos}^{\sin }(M, u)$ by $[g]$.

Proposition 2.4. Let $M$ be a spin manifold with fundamental group $\Gamma$, of dimension $m \geq 5$. If $[g] \in \operatorname{charPos}^{\mathrm{spin}}(M, u) \subset \operatorname{Pos}_{m}^{\mathrm{spin}}(B \Gamma)$, and $x \in \operatorname{ker}\left(\operatorname{Pos}_{m}^{\mathrm{spin}}(B \Gamma) \rightarrow\right.$ $\left.\Omega_{m}^{\mathrm{spin}}(B \Gamma)\right)$, then $x+[g] \in \operatorname{charPos}^{\mathrm{spin}}(M, u)$. The action of $\operatorname{ker}\left(\operatorname{Pos}_{m}^{\mathrm{spin}}(B \Gamma) \rightarrow\right.$ $\left.\Omega_{m}^{\text {spin }}(B \Gamma)\right)$ on charPos ${ }^{\text {spin }}(M, u)$ is free and transitive.

Proof. The statement is a consequence of the surgery result of Gromov-Lawson, Schoen-Yau, Gajer (compare [Botvinnik and Gilkey 1995, Lemma 3.1]). Since the underlying manifold $(X, f: X \rightarrow B \Gamma)$ of $x$ is zero bordant in $\Omega_{m}^{\text {spin }}(B \Gamma)$, the sum of $(M, u)$ and $(X, f)$ is bordant to $(M, u)$ in $\Omega_{m}^{\text {spin }}(B \Gamma)$. By assumption, on 
this sum we have a metric with scal $>0$. This metric can, by the surgery result, be extended over a suitable modification of the interior of this bordism (we have to make it sufficiently connected) to yield some metric with scal $>0$ on the other end, that is, on $(M, u)$. If we perform two such constructions, we can glue the resulting bordisms (with their metric and reference map to $B \Gamma$ ) along the boundary $(M+X, u+f)$ to see that the bordism class of the resulting metric is well defined.

Since the action comes from addition in $\operatorname{Pos}_{n}^{\text {spin }}(B \Gamma)$, the statement about freeness follows immediately. To prove transitivity, we simply observe that any two objects $\left[g_{1}\right],\left[g_{2}\right] \in \operatorname{charPos}^{\text {spin }}(M, u)$ map to the same element of $\Omega_{n}^{\text {spin }}(B \Gamma)$, so that their difference belongs to $\operatorname{ker}\left(\operatorname{Pos}_{m}^{\text {spin }}(B \Gamma) \rightarrow \Omega_{m}^{\text {spin }}(B \Gamma)\right)$. Thus

$\left[g_{2}\right]=\left[g_{1}\right]+\left(\left[g_{2}\right]-\left[g_{1}\right]\right)=\left[g_{1}\right]+x$, with $x \in \operatorname{ker}\left(\operatorname{Pos}_{m}^{\mathrm{spin}}(B \Gamma) \rightarrow \Omega_{m}^{\mathrm{spin}}(B \Gamma)\right)$, and we are done.

Now, we want to introduce invariants on $\operatorname{Pos}_{m}^{\text {spin }}(B \Gamma)$ and $\operatorname{charPos}^{\mathrm{spin}}(M, u)$ that can be used to distinguish elements in these sets.

Definition 2.5. Let $(M, g)$ be a spin manifold with Riemannian metric $g$ and with reference map $u: M \rightarrow B \Gamma$. Let $\bar{M}$ be the $\Gamma$-covering classified by $u$ (if $u$ is a $\pi_{1}$-isomorphism, then $\bar{M}$ is a universal covering of $M$ ). Define

$$
\rho_{(2)}(M, g, u):=\eta_{(2)}(\bar{D})-\eta(D),
$$

where $D$ is the spin Dirac operator on $M$ and $\bar{D}$ its lift to $\bar{M}$. For details on the eta and the $L^{2}$-eta invariant, compare, for example, [Piazza and Schick 2007].

Fix an element $h \in \Gamma$ such that its conjugacy class $<h>$ has polynomial growth (inside $\Gamma$ with its word metric). If the scalar curvature of $(M, g)$ is strictly positive, then the Dirac operator of $M$ (and the Dirac operator twisted with any flat bundle) is invertible. Consequently, the delocalized eta invariant of Lott, denoted $\eta_{<h>}(\bar{D})$, is defined (compare [Lott 1999; 2004]; see also [Piazza and Schick 2007, Section 13.1]). More precisely,

$$
\eta_{<h>}(\bar{D})=\frac{1}{\sqrt{\pi}} \int_{0}^{\infty} \sum_{\gamma \in<h>}\left(\int_{\mathscr{F}} \operatorname{tr}_{x} k_{t}(x, \gamma x) d x\right) \frac{d t}{\sqrt{t}},
$$

here $k_{t}(x, y)$ is the integral kernel of the operator $\bar{D} e^{-t \bar{D}^{2}}$ on the covering $\bar{M}:=$ $u^{*} E \Gamma$, and $\mathscr{F}$ is a fundamental domain for this covering. Note that it is a highly nontrivial fact that this sum and integral converge; it is proved for invertible $\bar{D}$ and groups $\Gamma$ of polynomial growth in [Lott 1999]; we observed in [Piazza and Schick 2007] that one can take arbitrary groups, provided the conjugacy class is of polynomial growth. Some information about conjugacy classes of polynomial growth can be found in [Wiethaup 2004]. We give an example where the expression 
does not converge in Section 3. Notice that the same formula, if $h=1$, defines $\eta_{(2)}(\bar{D})$.

Notation 2.6. If $h \neq 1$ we shall set $\rho_{<h>}(M, g, u):=\eta_{<h>}(\bar{D})$.

Proposition 2.7. The invariants $\rho_{(2)}$ and $\rho_{<h>}$ of Definition 2.5 define homomorphisms

$$
\rho_{(2)}: \operatorname{Pos}_{*}^{\text {spin }}(B \Gamma) \rightarrow \mathbb{R}, \quad \rho_{<h>}: \operatorname{Pos}_{*}^{\text {spin }}(B \Gamma) \rightarrow \mathbb{C} .
$$

Proof. The group structure in $\operatorname{Pos}_{m}^{\text {spin }}(B \Gamma)$ is given by disjoint union, and $\rho_{(2)}$ as well as $\rho_{<h>}$ are additive under disjoint union. We only have to show that the invariant vanishes for a manifold representing 0 in $\operatorname{Pos}_{m}^{\text {spin }}(B \Gamma)$. Similar arguments have already been used in many places, for example, [Botvinnik and Gilkey 1995, Theorem 1.1] and [Leichtnam and Piazza 2001, Proposition 4.1]. Let $[M, g, u]=0$ in $\operatorname{Pos}_{m}^{\text {spin }}(B \Gamma)$; then there exists a spin Riemannian manifold $(W, G)$ together with a classifying map $U: W \rightarrow B \Gamma$ such that $\partial W=M,\left.U\right|_{\partial W}=u, \operatorname{scal}(G)>0$, $\left.G\right|_{\partial W}=g$. Let $\mathscr{D}_{W}$ be the Mishchenko-Fomenko spin Dirac operator associated to $(W, G, U)$; let $C^{*} \Gamma$ be the maximal group $C^{*}$-algebra. Since scal $(g)>0$, there is a well-defined index class $\operatorname{Ind}\left(\mathscr{D}_{W}\right) \in K_{0}\left(C^{*} \Gamma\right)$; since $\operatorname{scal}(G)>0$ this index class is zero (the operator $\mathscr{D}_{W}$ is in fact invertible). Let $\operatorname{Ind}_{[0]}\left(\mathscr{D}_{W}\right):=\operatorname{Tr}^{\text {alg }}\left(\operatorname{Ind}\left(\mathscr{D}_{W}\right)\right)$ with $\operatorname{Tr}^{\text {alg }}: K_{0}\left(C^{*} \Gamma\right) \rightarrow C^{*} \Gamma / \overline{\left[C^{*} \Gamma, C^{*} \Gamma\right]}$ the natural algebraic trace. Then, the APS index theorem proved in [Leichtnam and Piazza 1997] (see [Piazza and Schick 2007, Theorem 3.3] for a direct and elementary proof of the special case used here) gives

$$
0=\operatorname{Ind}_{[0]}\left(\mathscr{D}_{W}\right)=\left(\int_{W} \widehat{A}(W, G)\right) \cdot 1-\frac{1}{2} \eta_{[0]}\left(\mathscr{D}_{M}\right) \in C^{*} \Gamma / \overline{\left[C^{*} \Gamma, C^{*} \Gamma\right]}
$$

a similar identity holds in the abelianization $C_{r}^{*} \Gamma / \overline{\left[C_{r}^{*} \Gamma, C_{r}^{*} \Gamma\right]}$ of the reduced group $C^{*}$-algebra as well as in the abelianization of the Connes-Moscovici algebra $\mathscr{B}_{\Gamma}^{\infty}$ :

$$
0=\operatorname{Ind}_{[0]}\left(\mathscr{D}_{W}^{\infty}\right)=\left(\int_{W} \widehat{A}(W, G)\right) \cdot 1-\frac{1}{2} \eta_{[0]}\left(\mathscr{D}_{M}^{\infty}\right) \in \mathscr{B}_{\Gamma}^{\infty} / \overline{\left[\mathscr{B}_{\Gamma}^{\infty}, \mathscr{P}_{\Gamma}^{\infty}\right]} .
$$

Let $h \neq 0$ and $\tau_{<h>}: \mathbb{C} \Gamma \rightarrow \mathbb{C}$ be the trace defined by $\sum_{g \in \Gamma} \lambda_{g} g \mapsto \sum_{g \in<h>} \lambda_{g}$. Because the conjugacy class $\langle h\rangle$ has polynomial growth, we observed in [Piazza and Schick 2007, Proposition 13.5] that $\tau_{<h>}$ extends by continuity to a trace on $\mathscr{B}_{\Gamma}^{\infty}$. By [Lott 1999, Formula (4.16)],

$$
\tau_{<h>}\left(\eta_{[0]}\left(\mathscr{D}_{M}^{\infty}\right)\right)=\eta_{<h>}(\bar{D}) \equiv \rho_{<h>}(M, g, u),
$$

and since $\tau_{<h>}(1)=0$, we finally see that by applying $\tau_{<h>}$ to (2.9) we get $\rho_{<h>}(M, g, u)=0$, which is what we wanted to prove.

Let $\tau: C^{*} \Gamma \rightarrow \mathbb{C}$ be the trace induced by the trivial representation; let $\tau_{\Gamma}: C^{*} \Gamma \rightarrow$ $\mathbb{C}$ be the canonical trace, that is, the trace induced by $\sum_{g \in \Gamma} \lambda_{g} g \mapsto \lambda_{1}$. Obviously 
$\tau(1)=\tau_{\Gamma}(1)$. Recall now that we have also proved in [Piazza and Schick 2007] that

$$
\tau_{\Gamma}\left(\eta_{[0]}\left(\mathscr{D}_{M}\right)\right)=\eta_{(2)}(\bar{D}), \quad \tau\left(\eta_{[0]}\left(\mathscr{D}_{M}\right)\right)=\eta(D) ;
$$

we complete the proof by applying $\tau$ and $\tau_{\Gamma}$ to (2.8) and subtracting.

Proposition 2.10. Let $\mathscr{R}^{+}(M)$ be the space of smooth metrics with positive scalar curvature on $M$, with the usual $C^{\infty}$-topology. For the fixed spin structure and classifying map $u: M \rightarrow B \Gamma=B \pi_{1}(M)$, we get an obvious surjection $\mathscr{R}^{+}(M) \rightarrow$ $\operatorname{charPos}^{\text {spin }}(M, u)$. The composition

$$
\mathscr{R}^{+}(M) \rightarrow \operatorname{charPos}^{\text {spin }}(M, u) \hookrightarrow \operatorname{Pos}_{m}^{\text {spin }}(B \Gamma) \stackrel{\rho_{(2)}}{\longrightarrow} \mathbb{R}
$$

is constant on orbits of the action of the spin-structure-preserving diffeomoprhisms $\operatorname{Diffeo\# }(M)$ (which acts by pulling back the Riemannian metric). Moreover, it is locally constant, and therefore factors through the set of components of the moduli space $\pi_{0}\left(\Re^{+}(M) / \operatorname{Diffeo\# }_{\#}(M)\right)$.

Proof. Let PSpin $(T M)$ be a 2-fold covering of $\mathrm{PSO}_{g}(T M) \rightarrow M$ that is nontrivial on the fibers and that determines the chosen spin structure on $M$. Equivalence of spin structures is understood as equivalence of such 2-fold coverings. Using that the inclusion $\mathrm{PSO}_{h}(T M) \hookrightarrow \mathrm{PGL}_{+}(T M)$ is a homotopy equivalence for each metric $h$ on $T M$, we can equivalently define a spin structure as a 2-fold covering of $\mathrm{PGL}_{+}(T M)$ that is nontrivial along the fibers of $\mathrm{PGL}_{+}(T M) \rightarrow M$; this means, in particular, that the choice of a spin structure for one metric $g$ canonically determines a spin structure for any other metric $h$-compare [Lawson and Michelsohn 1989, Chapter II, Sections 1 and 2]. Let $\Psi: M \rightarrow M$ be a diffeomorphism; let $d \Psi: \mathrm{PGL}_{+}(T M) \rightarrow \mathrm{PGL}_{+}(T M)$ be the induced diffeomorphism. Then $\Psi$ is spin structure preserving if the pullback $d \Psi^{*}(\operatorname{PSpin}(T M))$ is equivalent to $\operatorname{PSpin}(T M)$. Call the corresponding isomorphism $\beta_{G L_{+}}$. Now, if we define the spinor bundle, $L^{2}$-spinors and the Dirac operator entirely in terms of the pullback structures, $\Psi$ induces a unitary equivalence, and consequently the eta invariant of $D$ and of the operator defined using the pulled back structure coincide. On the other hand, the isomorphism $\beta_{G L_{+}}$induces an isomorphism $\beta$ between the original spin structure and the pulled-back structure, where both are seen as twofold coverings of $\mathrm{PSO}_{\Psi^{*} g}(T M)$; using $\beta$ we get a unitary equivalence between the operator obtained via the pulled back structures and the Dirac operator for $\Psi^{*} g$ and the chosen fixed spin structure, so that their eta invariants coincide, as well. Taken together, $\eta\left(D_{g}\right)=\eta\left(D_{\Psi^{*} g}\right)$. More or less, the same applies to the construction of the $L^{2}$ eta invariant on the universal covering. To simplify the notation, let us denote by $P$ the chosen spin structure. We start with a given covering $\bar{M} \stackrel{\pi}{\rightarrow} M$ with given action of $\Gamma$ by deck transformations (obtained by pulling back $E \Gamma$ from $B \Gamma$ via the map $u: M \rightarrow B \Gamma)$. The spin structure and the metric on $\bar{M}$, denoted by $\bar{g}$ 
and $\bar{P}$, are the ones pulled back from $M$ via $\pi$. We can then pull back everything, including the covering $\bar{M}$ via $\Psi$, and will obtain a $\Gamma$-covering $p: \Psi^{*} \bar{M} \rightarrow M$ with pullback $\Gamma$-action, spin structure, pullback metric, and so on. Then $\Psi$ will induce a unitary $\Gamma$-equivariant equivalence between $\bar{D}$ and the Dirac operator constructed entirely in terms of the pulled back structures; so the $L^{2}$-eta invariants of these two operators coincide. On the other hand, we have the covering $\bar{M}$ itself and the fixed spin structure. Since the universal covering is unique, we get a covering isomorphism $\gamma: \bar{M} \rightarrow \Psi^{*} \bar{M}$ covering the identity. It becomes an isometry if we use on $\bar{M}$ the lift of the metric $\Psi^{*} g$. On $\Psi^{*} \bar{M}$ we have used the spin structure given by the pullback principle bundle $\bar{\Psi}^{*} \bar{P}$, with $\bar{\Psi}$ the obvious map $\Psi^{*} \bar{M} \rightarrow \bar{M}$ covering $\Psi$. Since $p \circ \gamma=\pi$, we get a map of principal bundles

$$
\pi^{*} \Psi^{*} P \rightarrow \bar{\Psi}^{*} \bar{P}
$$

We now use the principal bundle isomorphism $P \rightarrow \Psi^{*} P$, which comes from the fact that $\Psi$ is spin structure preserving, to finally identify the spin principle bundle of $\bar{M}$ to the one of $\Psi^{*} \bar{M}$ via a map $\bar{\gamma}$ covering $\gamma$ and the map $P \rightarrow$ $\Psi^{*} P$ of principal bundles on $M$. Proceeding as for $M$ itself, we obtain a unitary equivalence between $\bar{D}_{\Psi^{*} g}$ and the operator obtained using the pullback structures. Summarizing: $\eta_{(2)}\left(\bar{D}_{g}\right)=\eta_{(2)}\left(\bar{D}_{\Psi^{*} g}\right)$.

Remark 2.11. The map $\gamma$ is not, in general, $\Gamma$-equivariant, but we can choose $\gamma$ so that for $x \in \bar{M}$ and $g \in \Gamma, \gamma(g x)=\alpha_{\Psi}(g) \gamma(x)$, where $\alpha_{\Psi}: \Gamma \rightarrow \Gamma$ is equal to the isomorphism $u_{*} \pi_{1}(\Psi) u_{*}^{-1}$. This is because (by the universal property of $B \Gamma$ and $E \Gamma), \Psi^{*} u^{*} E \Gamma$ is isomorphic as $\Gamma$-principal bundle to $u^{*}\left(B \alpha_{\Psi}\right)^{*} E \Gamma$, since $u \circ \Psi$ and $B \alpha_{\Psi} \circ u$ induce the same map on the fundamental group. Moreover, by [Lawson and Michelsohn 1989, Appendix B, p. 378], $\left(B \alpha_{\Psi}\right)^{*} E \Gamma$ is isomorphic as $\Gamma$-principal bundle to the associated bundle $\Gamma \times_{\alpha_{\Psi}} E \Gamma$, and the required covering isomorphism

$$
E \Gamma \rightarrow \Gamma \times_{\alpha_{\Psi}} E \Gamma ; x \mapsto[1, x]
$$

has exactly the required equivariance property:

$$
[1, g x]=\left[\alpha_{\Psi}(g), x\right]=\alpha_{\Psi}(g)[1, x]
$$

which is preserved when pulling back the whole covering isomorphism with $u$.

Now, as explained above, the map $\gamma$ induces maps which preserve all the structure present in the construction of the Dirac operators on $\bar{M}$ (using the lift of the metric $\Psi^{*} g$ ) and $\Psi^{*} \bar{M}$ (except for the group action). In particular, for the fiberwise trace, we have

$$
\operatorname{tr} k_{t}(x, h x)=\operatorname{tr} \kappa_{t}\left(\gamma(x), \alpha_{\Psi}(h) \gamma(x)\right)
$$


where here $k_{t}(x, y)$ is the integral kernel of $\bar{D} e^{-t \bar{D}^{2}}$ on $\bar{M}$ using the fixed spin structure and the metric $\Psi^{*} g$, whereas $\kappa_{t}(x, y)$ is the same function on $\Psi^{*} \bar{M}$ defined using the pullback structure throughout.

In particular, reasoning as in the proof of Proposition 2.10, we see that for $h \in \Gamma$

$$
\eta_{<h>}\left(\bar{D}_{\Psi^{*} g}\right)=\eta_{<\alpha_{\Psi}(h)>}(\bar{D}),
$$

whenever $\eta_{<h>}$ is defined.

The following example is a direct consequence of the results of Botvinnik and Gilkey [Gilkey 1984].

Example 2.13. Let $\mathbb{Z} / n$ be a finite cyclic group and $m>4$ be congruent $3 \bmod 4$. Then $\rho_{(2)}: \operatorname{Pos}_{m}^{\text {spin }}(B \mathbb{Z} / n) \rightarrow \mathbb{R}$ is nontrivial. Since it is a group homomorphism for the additive group of $\mathbb{R}$, its image is infinite.

Proof. We only have to observe that $\rho_{(2)}$ is a twisted rho invariant, where we twist with $-\mathbb{R}+\frac{1}{n} \mathbb{R}[\mathbb{Z} / n]$. Indeed, the first representation is the opposite of the trivial representation, giving minus the ordinary eta invariant; the second one is a multiple of the regular representation, giving the $L^{2}$-eta invariant. To prove the last statement, recall that for any unitary representation $\phi$ with character $\chi_{\phi}$, the twisted eta invariant $\eta_{\phi}(D)$ can be expressed by

$$
\eta_{\phi}(D)=\sum_{h \in \mathbb{Z} / n} \chi_{\phi}(h) \eta_{h}(\bar{D}),
$$

where $<h>=h$, given that the group is commutative. Since the character of the regular representation is the delta function at the identity element, we see that the eta invariant for the operator twisted by the regular representation is nothing but the $\eta$-invariant of the $\mathbb{Z} / n$-covering, which is $n$-times the $L^{2}$-eta invariant of this covering.

The character $\chi$ of the virtual representation $-\mathbb{R}+1 / n \mathbb{R}[\mathbb{Z} / n]$ is invariant under inversion: $\chi(g)=\chi\left(g^{-1}\right)$. This means, by definition, that $-\mathbb{R}+\frac{1}{n} \mathbb{R}[\mathbb{Z} / n] \in$ $R_{0}^{+}(\mathbb{Z} / n)$, where

$$
R_{0}^{+}(\mathbb{Z} / n):=\left\{\phi \in R(\mathbb{Z} / n) \mid \chi_{\phi}(1)=0 ; \chi_{\phi}(g)=\chi_{\phi}\left(g^{-1}\right) \text { for all } g\right\} .
$$

By the results of Botvinnik and Gilkey [1995, Proof of Theorem 2.1] we know

$$
\text { for all } \phi \in R_{0}^{+}(\mathbb{Z} / n) \text {, there exists }[M, g, u] \in \operatorname{Pos}_{m}^{\text {spin }}(B \mathbb{Z} / n)
$$
such that $\rho_{\phi}[M, g, u] \neq 0$,

and it suffices to apply this result to $\mathbb{R}-\frac{1}{n} \mathbb{R}[\mathbb{Z} / n]$. 
Remark 2.17. Let $\Gamma$ be any finite group and let $m>4$ be congruent $3 \bmod 4$. The results of Botvinnik and Gilkey show that the map $\Psi: R_{0}^{+}(\Gamma) \otimes \mathbb{C} \rightarrow\left(\operatorname{Pos}_{m}^{\text {spin }}(B \Gamma) \otimes\right.$ $\mathbb{C})^{\prime}$ defined by

$$
\Psi(\phi)[M, g, u]:=\rho_{\phi}[M, g, u]
$$

is injective. Let Class $(\Gamma)=\left\{f: \Gamma \rightarrow \mathbb{C} \mid f\left(\gamma^{-1} h \gamma\right)=f(h)\right.$, for all $\left.\gamma, h \in \Gamma\right\}$ be the complex vector space of class functions on $\Gamma$. Let

$$
\operatorname{Class}_{0}^{+}(\Gamma)=\left\{f \in \operatorname{Class}(\Gamma) \mid f(1)=0, f(h)=f\left(h^{-1}\right)\right\} .
$$

Then there is a natural isomorphism of vector spaces $\Theta: R_{0}^{+}(\Gamma) \otimes \mathbb{C} \rightarrow \mathrm{Class}_{0}^{+}(\Gamma)$ obtained by associating to $\phi \in R_{0}^{+}(\Gamma)$ its character $\chi_{\phi}$. There is also a map $\Phi$ : $\operatorname{Class}_{0}^{+}(\Gamma) \rightarrow\left(\operatorname{Pos}_{m}^{\text {spin }}(B \Gamma) \otimes \mathbb{C}\right)^{\prime}$ given by

$$
\Phi(f)[M, g, u]:=\sum_{<h>} \rho_{<h>}[M, g, u] f(<h>) .
$$

Since by the analog of (2.14) we see that $\Phi \circ \Theta=\Psi$, we conclude that $\Phi$ is also injective if $m>4$ is congruent $3 \bmod 4$.

We shall apply this result to $\Gamma=\mathbb{Z} / n$ : thus, for these values of $m$,

$$
\begin{aligned}
& \text { for all } f \in \operatorname{Class}_{0}^{+}(\mathbb{Z} / n) \text {, there exists } y \in \operatorname{Pos}_{m}^{\text {spin }}(B \mathbb{Z} / n) \otimes \mathbb{C} \\
& \text { such that } \sum_{h} \rho_{h}(y) f(h) \neq 0 .
\end{aligned}
$$

The following lemma describes how to compute delocalized rho invariants for manifolds obtained by induction.

Lemma 2.22. Let $\pi=\mathbb{Z} / n$ be a finite cyclic group and $j: \mathbb{Z} / n \hookrightarrow \Gamma$ be an injective group homomorphism. We then have the induced map $B j_{*}: \operatorname{Pos}_{m}^{\text {spin }}(B \mathbb{Z} / n) \rightarrow$ $\operatorname{Pos}_{m}^{\text {spin }}(B \Gamma)$. Fix $1 \neq g \in \Gamma$ of finite order and such that its conjugacy class $<g>$ has polynomial growth. The delocalized rho invariant $\rho_{<g>}: \operatorname{Pos}_{m}^{\text {spin }}(B \Gamma) \rightarrow \mathbb{R}$ is defined if $m$ is odd. Then

$$
\rho_{<g>}\left(B j_{*} x\right)=\sum_{h \in j^{-1}(<g>)} \rho_{<h>}(x), \quad \text { for all } x \in \operatorname{Pos}_{m}^{\mathrm{spin}}(B \mathbb{Z} / n) .
$$

Similarly, considering the $L^{2}$-rho invariant,

$$
\rho_{(2)}\left(B j_{*} x\right)=\rho_{(2)}(x), \quad \text { for all } x \in \operatorname{Pos}_{m}^{\mathrm{spin}}(B \mathbb{Z} / n) .
$$

Proof. This is a well-known feature of $L^{2}$-invariants. We indicate the proof, showing along the way how it extends to the delocalized invariants. Assume that $x=[M, g, u: M \rightarrow B \pi]$. Observe that $j$ is injective. This implies that the covering $(B j)^{*} E \Gamma \rightarrow B \pi$ decomposes as a disjoint union (parametrized by the elements of the set $\Gamma / j(\pi))$ of copies of $E \pi$. For the convenience of the reader, we recall 
a possible argument. Given the universal free $\Gamma$-space $E \Gamma$, the action of $\pi$ on $E \Gamma$ (via $j$ ) allows us to view $E \Gamma$ as a model of $E \pi$, with $B \pi:=E \Gamma / \pi$. In this picture, $B j$ is simply the projection map $E \Gamma / \pi \rightarrow E \Gamma / \Gamma$. Then the pullback $(B j)^{*} E \Gamma=\{(x \pi, x \gamma) \in E \Gamma / \pi \times E \Gamma \mid x \pi \in E \Gamma / \pi, \gamma \in \Gamma\} \cong E \Gamma \times \pi \backslash \Gamma$, with the evident map $(x \pi, x \gamma) \mapsto(x \pi, \pi \gamma)$.

Consequently, the covering $\bar{M}=(B j \circ u)^{*} E \Gamma=u^{*}(B j)^{*} E \Gamma$ decomposes as a disjoint union of copies of the covering $\tilde{M}$ classified by $u$. The construction of the $L^{2}$-eta invariant for this disjoint union $\bar{M}=(B j \circ u)^{*} E \Gamma$ involves only the one component $\tilde{M}$ that contains the fundamental domain, and therefore is exactly the same as the construction of the $L^{2}$-eta invariant for $\tilde{M}$ itself. Since the ordinary $\eta$-invariant does only depend on $M$, also the $L^{2}$-rho invariants coincide.

More precisely, and also holding for the delocalized invariants, recall from Definition 2.5 that

$$
\rho_{<h>}(\bar{D})=\frac{1}{\sqrt{\pi}} \int_{0}^{\infty} \sum_{\gamma \in<h>} \int_{\mathscr{F}} \operatorname{tr}_{x} k_{t}(x, \gamma x) d x \frac{d t}{\sqrt{t}} .
$$

Now $\bar{M}$ decomposes as a disjoint union of copies of $\tilde{M}$. The heat kernel $k_{t}(x, y)$ vanishes if $x$ and $y$ belong to different components and, if $x$ and $y$ lie in the same component, coincides with the heat kernel of the operator restricted to that component (use uniqueness of the heat kernel). If $x \in \mathscr{F} \subset \tilde{M}$ and $\gamma \in \operatorname{im}(j)$ then $\gamma x \in \tilde{M}$ (because $\tilde{M} \rightarrow M$ is just the covering corresponding to the subgroup $j(\pi)$ of $\Gamma$ ). However, if $\gamma \notin \operatorname{im}(j)$, then $\gamma x \notin \tilde{M}$ (for the same reason). Thus, in the sum above, all summands with $\gamma \notin \operatorname{im}(j)$ vanish, whereas the summands with $\gamma \in \operatorname{im}(j)$ are exactly those (using an obvious diffeomorphism) showing up in the definition of the delocalized invariants for $\tilde{D}$ on $\tilde{M}$.

Remark 2.24. The proof of Lemma 2.22 gives also a formula for induction from arbitrary (not necessarily cyclic) subgroups. Namely, if $j: \pi \hookrightarrow \Gamma$ is an injective homomorphism

$$
\rho_{<h>}\left(B j_{*}(x)\right)=\sum_{<\gamma>\subset j^{-1}(<h>)} \rho_{<\gamma>}(x),
$$

where the sum on the right hand side runs over all the $\pi$-conjugacy classes that are contained in $j^{-1}(<h>)$.

Theorem 2.25. Assume that $M$ is a spin manifold of dimension $m>4, m \equiv 3$ (mod 4). Assume that $g$ is a metric with positive scalar curvature on $M$, and that the fundamental group $\Gamma$ of $M$ contains at least one nontrivial element of finite order. Then charPos ${ }^{\operatorname{spin}}(M, u)$ is infinite, that is, $M$ admits infinitely many different bordism classes of metric with $\mathrm{scal}>0$. They are distinguished by $\rho_{(2)}$. 
More precisely, the infinitely many bordism classes we construct are also different modulo the "action" of the diffeomorphism group, that is, we get metrics $\left(g_{\alpha}\right)_{\alpha \in A}$ such that $|A|=\infty$, and for every diffeomorphism $f$ of $M, f^{*} g_{\alpha}$ is bordant to $g_{\beta}$ only if $\alpha=\beta$.

As a consequence, the space $\mathscr{R}^{+}(M) / \operatorname{Diffeo}(M)$, the moduli space of metrics of positive scalar curvature, has infinitely many components, distinguished by $\rho_{(2)}$.

Remark 2.26. Recall that by the methods of Gromov and Lawson, it is known that $\mathscr{R}^{+}(M) / \operatorname{Diffeo}(M)$ has infinitely many components for every manifold of dimension $4 k+3, k \geq 1$ (compare [Lawson and Michelsohn 1989, Theorem 7.7]). Strictly speaking the result stated there only involves $\mathscr{R}^{+}(M)$ : an inspection of the proof shows that the main argument also establishes that $\left|\pi_{0}\left(\mathscr{R}^{+}(M) / \operatorname{Diffeo}(M)\right)\right|$ $=\infty$ : indeed it suffices to observe that the signature is a cut-and-paste invariant. Notice, however, that by construction the examples they get are all bordant to each other.

Proof. Let $j: \mathbb{Z} / n \rightarrow \Gamma$ be an injection. This exists for some $n>1$ since $\Gamma$ is not torsion free. By Example 2.13, the homomorphism $\rho_{(2)}: \operatorname{Pos}_{m}^{\text {spin }}(B \mathbb{Z} / n) \rightarrow \mathbb{R}$ is nontrivial (therefore has infinite image). The group $\Omega_{m}^{\text {spin }}(B \mathbb{Z} / n)$ is finite by the Atiyah-Hirzebruch spectral sequence. Consequently, the kernel $K$ of the map $\operatorname{Pos}_{m}^{\text {spin }}(B \mathbb{Z} / n) \rightarrow \Omega_{m}^{\text {spin }}(B \mathbb{Z} / n)$ has finite index, and the restriction $\rho_{(2)} \mid: K \rightarrow \mathbb{R}$ also is nontrivial with an infinite image.

Let $u: M \rightarrow B \Gamma$ be the chosen classifying map of a universal covering. By naturality of the exact sequence (2.1) and Proposition 2.4, $B j_{*} k+[M, g, u] \in$ $\operatorname{charPos}^{\operatorname{spin}}(M, u)$ for each $k \in K$. Moreover, by Lemma 2.22,

$$
\rho_{(2)}\left(B j_{*} k+[M, g, u]\right)=\rho_{(2)}(k)+\rho_{(2)}(M, g, u),
$$

Consequently, $\rho_{(2)}: \operatorname{charPos}^{\operatorname{spin}}(M, u) \rightarrow \mathbb{R}$ has an infinite image.

Using Proposition 2.10 and the surjectivity of $\mathscr{R}^{+}(M) \rightarrow \operatorname{charPos}^{\mathrm{spin}}(M, u)$, the map $\rho_{(2)}: \pi_{0}\left(\mathscr{R}^{+}(M) / \operatorname{Diffeo\# }(M)\right) \rightarrow \mathbb{R}$ also has an infinite image.

Since the spin-structure preserving diffeomorphisms have finite index in all diffeomorphisms, even modulo $\operatorname{Diffeo}(M)$, there are infinitely many components in the moduli space. In a similar way, we can get infinitely many bordism classes that are different even modulo pullback with arbitrary diffeomorphisms.

\section{B. Different conjugacy classes of torsion elements in the fundamental group} and positive scalar curvature. In this subsection, we shall sharpen Theorem 2.25 and extend it to dimensions $4 k+1$ under some additional assumptions on $\Gamma$.

Theorem 2.27. Let $\Gamma$ be a discrete group. Consider the following subset of the set $\mathscr{b}$ of all conjugacy classes of $\Gamma$ :

$$
\mathscr{C}_{f p}:=\{<h>\subset \Gamma \mid \text { h has finite order },<h>\text { has polynomial growth }\} .
$$


On this set, we have an involution $\tau$ given by $<h>\mapsto<h^{-1}>$. Assume that $M$ is a closed spin manifold with fundamental group $\Gamma$, with classifying map $u: M \rightarrow B \Gamma$, of dimension $4 k+3, k \geq 1$. Then, on $\operatorname{charPos}^{\operatorname{spin}}(M, u)$, a free abelian group of rank $\left|\mathscr{C}_{f p} / \tau\right|$ acts freely.

Proof. Let $K:=\operatorname{ker}\left(\operatorname{Pos}_{m}^{\text {spin }}(B \Gamma) \rightarrow \Omega_{m}^{\text {spin }}(B \Gamma)\right)$ : then it suffices to show that $\operatorname{dim} K \otimes \mathbb{C} \geq\left|\mathscr{C}_{f p} / \tau\right|$. For each $<g>\in \mathscr{C}_{f p}$ consider the characteristic function $\kappa(g)$ of the set $<g>\cup<g^{-1}>$. By (2.19), $\kappa(g)$ belongs to Class ${ }_{0}^{+}(\Gamma)$. Let $L_{f p}$ be the vector subspace of $\mathrm{Class}_{0}^{+}(\Gamma)$ whose elements are finite linear combinations of $\kappa(g)$, with $<g>\in \mathscr{C}_{f p}$. This is a vector space of dimension $\left|\mathscr{C}_{f p} / \tau\right|$, and we denote by $\kappa, \kappa=\sum_{j} \lambda_{j} \kappa\left(g_{j}\right)$ the generic element. Following Remark 2.17, we begin by showing that the map $\Phi: L_{f p} \subset \operatorname{Class}_{0}^{+}(\Gamma) \rightarrow\left(\operatorname{Pos}_{m}^{\text {spin }}(B \Gamma) \otimes \mathbb{C}\right)^{\prime}$ that associates to $\kappa=\sum_{j} \lambda_{j} \kappa\left(g_{j}\right)$ the functional $\Phi(\kappa)$,

$$
\Phi(\kappa)[M, g, u]:=\sum_{j} \lambda_{j}\left(\rho_{<g_{j}>}[M, g, u]+\rho_{<g_{j}^{-1}>}[M, g, u]\right),
$$

is injective. Choose $g_{\ell}$ so that $\lambda_{\ell} \neq 0$. Let $\pi\left(g_{\ell}\right)$ be the finite cyclic group generated by $g_{\ell}$. Consider the restriction $\left.\kappa\right|_{\pi\left(g_{\ell}\right)}$, an element in $\operatorname{Class}_{0}^{+}\left(\pi\left(g_{\ell}\right)\right)$. Then by the results of Botvinnik-Gilkey, as stated in (2.21), we know that there exist $y \in$ $\operatorname{Pos}_{m}^{\text {spin }}\left(B \pi\left(g_{\ell}\right)\right) \otimes \mathbb{C}$ such that

$$
\left.\sum_{h \in \pi\left(g_{\ell}\right)} \rho_{h}(y) \kappa\right|_{\pi\left(g_{\ell}\right)}(h) \neq 0 .
$$

Let $j: \pi\left(g_{\ell}\right) \hookrightarrow \Gamma$ be the natural inclusion, and let $x:=B j_{*}(y)$ so that $x \in$ $\operatorname{Pos}_{m}^{\text {spin }}(B \Gamma) \otimes \mathbb{C}$. By the induction formula (2.23) we know that

$$
\Phi(\kappa)(x)=\left.\sum_{h \in \pi\left(g_{\ell}\right)} \rho_{h}(y) \kappa\right|_{\pi\left(g_{\ell}\right)}(h),
$$

and we can therefore conclude that $\Phi(\kappa)(x) \neq 0$. It remains to show that we can choose $x \in \operatorname{ker}\left(\operatorname{Pos}_{m}^{\text {spin }}(B \Gamma) \rightarrow \Omega_{m}^{\text {spin }}(B \Gamma)\right) \otimes \mathbb{C}$. By naturality of the long exact sequence (2.1), it suffices to show that we can choose $y \in \operatorname{ker}\left(\operatorname{Pos}_{m}^{\text {spin }}\left(B \pi\left(g_{\ell}\right)\right) \rightarrow\right.$ $\left.\Omega_{m}^{\text {spin }}\left(B \pi\left(g_{\ell}\right)\right)\right) \otimes \mathbb{C}$. However, since $\Omega_{m}^{\text {spin }}\left(B \pi\left(g_{\ell}\right)\right)$ is finite, this is easily accomplished by taking a suitable multiple of the original $y$.

We now analyze the case $\operatorname{dim}(M) \equiv 1(\bmod 4)$. Let

$$
\begin{aligned}
& R_{0}^{-}(\mathbb{Z} / n):=\left\{\phi \in R(\mathbb{Z} / n) \mid \chi_{\phi}(1)=0 ; \chi_{\phi}(h)=-\chi_{\phi}\left(h^{-1}\right) \text { for all } h\right\} \\
& \operatorname{Class}_{0}^{-}(\Gamma)=\left\{f \in \operatorname{Class}(\Gamma) \mid f(1)=0, f(h)=-f\left(h^{-1}\right)\right\} .
\end{aligned}
$$

Then the results of Botvinnik and Gilkey [1995] imply that the analogs of (2.16) of Example 2.13 and of (2.21) of Remark 2.17 hold. For the readers's convenience 
explicitly restate the latter property in this new context:

$$
\begin{aligned}
& \text { for all } f \in \operatorname{Class}_{0}^{-}(\mathbb{Z} / n), \\
& \text { there exists } y \in \operatorname{Pos}_{m}^{\text {spin }}(B \mathbb{Z} / n) \otimes \mathbb{C} \text { such that } \sum_{h} \rho_{h}(y) f(h) \neq 0 .
\end{aligned}
$$

Theorem 2.32. Let $\Gamma$ be a discrete group. Let $\mathscr{C}_{f p}$ and $\tau: \mathscr{C}_{f p} \rightarrow \mathscr{C}_{f p}$ be as in the statement of Theorem 2.27. Let $\mathscr{C}_{f p}^{0}=\left\{<h>\in \mathscr{C}_{f p} \mid<h>\neq<h^{-1}>\right\}$. Assume that $M$ is a closed spin manifold with fundamental group $\Gamma$, with classifying map $u: M \rightarrow B \Gamma$, of dimension $4 k+1, k \geq 1$. Then, on $\operatorname{charPos}^{\operatorname{spin}}(M, u)$, a free abelian group of rank $\left|\mathscr{C}_{f p}^{0} / \tau\right|$ acts freely.

Moreover, if $\mathscr{C}_{f p}^{0}$ is not empty then there are infinitely many bordism classes which are different modulo the "action" of the diffeomorphism group as in Theorem 2.25. As a consequence, the space $\mathscr{R}^{+}(M) / \operatorname{Diffeo}(M)$, the moduli space of metrics of positive scalar curvature, has infinitely many components in our situation, distinguished by the collection $\rho_{<h>},<h>\in \mathscr{C}_{f p}^{0}$.

Proof. Let $<h>\in \mathscr{C}_{f p}^{0}$ and consider the function $\kappa(h)$ which is equal to 1 on $<h>$, equal to -1 on $\left\langle h^{-1}>\right.$, and 0 elsewhere; $\kappa(h)$ so defined is an element of $\operatorname{Class}_{0}^{-}(B \Gamma)$. Let $L^{0}$ be the vector subspace of $\operatorname{Class}_{0}^{-}(B \Gamma)$ whose elements are finite linear combinations of $\kappa(h)$, with $<h>\in \mathscr{C}_{f p}^{0}$. This is a vector space of dimension $\left|\mathscr{C}_{f p}^{0} / \tau\right|$. Using the induction formula and (2.31), the proof now proceeds as in the proof of Theorem 2.27.

If $\mathscr{C}_{f p}^{0}$ is not empty, choose the collection of functions $\kappa(h)$ of $L^{0}$ for $<h>\epsilon$ $\mathscr{C}_{f p}^{0}$. Note that $\kappa\left(h^{-1}\right)=-\kappa(h)$. Then, dualizing $\Phi$, we get a map

$$
\mathscr{R}^{+}(M) \rightarrow \operatorname{Pos}_{m}^{\text {spin }}(B \Gamma) \otimes \mathbb{C} \rightarrow \mathbb{C}_{f p}^{\mathscr{C}_{f p}^{0}} ;[M, g, u] \mapsto(\Phi(\kappa(h))(M, g, u))_{<h>\in \mathscr{C}_{f p}^{0}}
$$

with infinite image. If we had chosen one half of the functions $\kappa(h)$, forming a basis, the map would have been surjective.

Now, given a spin structure preserving diffeomorphism $\Psi: M \rightarrow M$ (with a given lift to the spin principal bundle), we get an induced automorphism $\alpha_{\Psi}$ of $\Gamma$ as in the proof of Proposition 2.10, and an induced permutation of $\mathscr{C}_{f p}^{0}$. Moreover, by (2.12),

$$
\Phi(\kappa(h))\left(M, \Psi^{*} g, u\right)=\Phi\left(\kappa\left(\alpha_{\Psi}(h)\right)\right)(M, g, u),
$$

so that we above map induces a well defined map

$$
\pi_{0}\left(\mathscr{R}^{+}(M) / \operatorname{DiffeO}_{\#}(M)\right) \rightarrow \mathbb{C}_{f p}^{\mathscr{C}_{f p}^{0}} / \Sigma,
$$

where we quotient the right hand side by the action of the permutation group, permuting the entries of the vector. Since this group is finite, the image still is infinite. 
Since the spin structure preserving diffeomorphisms have finite index in the whole diffeomorphism group, even $\pi_{0}\left(\Re^{+}(M) / \operatorname{Diffeo}(M)\right)$ is infinite.

Remark 2.33. Notice, in particular, that if $\operatorname{dim}(M) \equiv 1(\bmod 4)$ and if $\Gamma$ contains an element $g$ of finite order not conjugate to its inverse and such that the conjugacy class $<g>$ has polynomial growth, then a manifold $M$ as above admits infinitely many pairwise nonbordant metrics of positive scalar curvature. To our knowledge, this is the first such result of considerable generality.

Remark 2.34. We want to point out that there are many nontrivial examples of groups $\Gamma$, where $\mathscr{C}_{f p}^{0}$ is nonempty. In particular, this applies to:

(1) groups with a central element of odd order (here the relevant conjugacy class consists of one element). For an arbitrary group $H$ and a finite group $F$ (of odd order), all nontrivial elements of $F$ in $F \times H$ have this property.

(2) many groups with a nontrivial finite conjugacy center, consisting of elements of finite order. Such groups are, for example, obtained as extensions $1 \rightarrow$ $F \rightarrow G \rightarrow H \rightarrow 1$ with $F$ finite (and of odd order).

(3) groups of polynomial growth with elements of finite order (in this case, every conjugacy class has of course polynomial growth).

(4) the restricted wreath product $\left(\oplus_{k \in \mathbb{Z}} \mathbb{Z} / n\right) \rtimes \mathbb{Z}$ (if $n=2$ this is called the lamplighter group). This is a group of exponential growth, such that every element in the normal subgroup $\oplus_{k \in \mathbb{Z}} \mathbb{Z} / n$ has an infinite conjugacy class of polynomial growth.

Similar examples give rise to nonempty $\mathscr{C}_{f p}$.

\section{Further questions and open problems.}

(1) We study only $\pi_{1}$-bordism, which is necessary for our method, because it uses the common fundamental group throughout. Nonetheless, this concept is somewhat unnatural from a geometric point of view. It would be interesting to know whether our examples remain nonbordant if we talk about the most obvious simple definition of bordism of metrics of positive scalar curvature, or to find any examples that are not bordant in this weak sense.

(2) We get some information about the number of components of the space of metrics of positive scalar curvature. What else can be said about its topology and, in particular, about higher homotopy groups?

(3) We prove that for spin manifolds of dimension $4 k+1$ with positive scalar curvature and with fundamental group containing a central element of odd order, the moduli space of metrics of positive scalar curvature has infinitely many components. In dimension $4 k+3$ this is known unconditionally - what about the given dimension $4 k+1$ ? 


\section{An example of a nonconvergent delocalized eta invariant}

In this section we compute Lott's delocalized $\eta$-invariant of an easy example and use this to produce an example where it does not converge.

Consider the manifold $S^{1}$ with the usual metric. The Dirac (and signature) operator of $S^{1}$ is (unitarily equivalent to) the operator $D=-i d / d x$.

The integral kernel $k_{t}(x, y)$ of $\tilde{D} \exp \left(-t \tilde{D}^{2}\right)$ on the universal covering $\mathbb{R}$ of $S^{1}$ is

$$
k_{t}(x, y)=-i \frac{x-y}{2 t \sqrt{2 \pi t}} \exp \frac{(x-y)^{2}}{4 t} .
$$

Fixing the fundamental domain $\mathscr{F}=[0,1]$ for the covering projection, and using the action by the deck transformation group $\mathbb{Z}$ by addition, $(x, n) \mapsto x+n$, the delocalized eta invariant for a subset $X \subset \mathbb{N}$ formally would be

$$
\begin{aligned}
\eta_{X}(\tilde{D}) & :=\frac{1}{\sqrt{\pi}} \int_{0}^{\infty} \sum_{n \in X} \int_{\mathscr{F}} k_{t}(x, x+n) d x \frac{d t}{\sqrt{t}} \\
& =-\frac{i}{4 \pi} \int_{0}^{\infty} \sum_{n \in X} \int_{0}^{1} \frac{n}{t^{2}} e^{n^{2} / 4 t} d x d t=-\frac{i}{4 \pi} \sum_{n \in X} \frac{1}{n} \int_{0}^{\infty} \frac{e^{1 / 4 t}}{t^{2}} d t,
\end{aligned}
$$

where at the end we use the substitution $t / n^{2}=s$ and that the integrands are all positive, so that we can interchange the summation over $n \in X \subset \mathbb{N}$ and the integral over $t$.

It is clear that this expression is divergent for suitable infinite $X \subset \mathbb{N}$.

Consider next the group $\Gamma=\mathbb{Q} \rtimes\left(\bigoplus_{n \in \mathbb{Z}} \mathbb{Z}\right)$, where the generator of the $n$-th summand of $\bigoplus \mathbb{Z}$ acts by multiplication with the $|n|$-th prime number. By the definition of semidirect products, the conjugacy class of $1 \in \mathbb{Q}$ in the kernel group is exactly $\mathbb{Q}_{>0}$. Its intersection with the subgroup $\mathbb{Z}$ generated by 1 is therefore $\mathbb{N}_{>0} \subset \mathbb{Z}$.

Consider in addition $G:=\Gamma \rtimes_{\alpha}$, the HNN-extension of $\Gamma$ along $\alpha: \bigoplus_{n \in \mathbb{Z}} \mathbb{Z} \rightarrow$ $\bigoplus_{n \in \mathbb{Z}} \mathbb{Z} ;\left(n \mapsto \lambda_{n}\right) \mapsto\left(n \mapsto \lambda_{n+1}\right)$, the shift of the nonnormal subgroup $\bigoplus_{n \in \mathbb{Z}} \mathbb{Z}$. Then $G$ is generated by 3 elements: 1 in the additive groups of $\mathbb{Q}$, a generator of the copy of $\mathbb{Z}$ labelled with zero in $\bigoplus_{n \in \mathbb{Z}} \mathbb{Z}$, and the stable letter $t$. Moreover, using the normal form of elements in an HNN-extension, the intersection of the conjugacy class of 1 with $\mathbb{Q}$ still consists of $\mathbb{Q}_{>0}$, and therefore the interesection with the additive subgroup of integers consists of the natural numbers.

Observe that $G$ is finitely generated, but by its definition only recursively countably presented. As such, by a standard procedure, $G$ can be embedded into a finitely presented group $H$ which is obtained as follows (compare [Rotman 1995, Theorem 12.18]). 
One first constructs an auxiliary group $B_{2}$, then considers the group $B_{3}=B_{2} * G$, the free product of $B_{2}$ and $G$. The next group is an HNN-extension of $B_{3}$ along a subgroup which is of the form $U * G$ for a suitable subgroup $U$ of $B_{2}$. The stable letters act trivially on $G$. By the normal form of elements of an HNN-extension, it follows that for every element $x \in G$, the conjugacy class of $x$ in $G$ is equal to the intersection of the conjugacy class of $x$ in $B_{3}$ with $G$.

In the next steps, one constructs two further HNN-extensions of the previous group (starting with $B_{3}$ ) along subgroups with trivial intersection with $G$. Again, it follows from the normal form of elements in an $\mathrm{HNN}$-extension that for every $x \in G$ the conjugacy class of $x$ in $G$ coincides with the intersection of $G$ with the conjugacy class of $x$ in the bigger group. The final group $H:=B_{6}$ is finitely presented, contains $G$ (and therefore $\mathbb{Z}$ ) as a subgroup, and the intersection of the conjugacy class of 1 with $\mathbb{Z}$ consists exactly of the positive integers.

Consider $u: S^{1} \rightarrow B \mathbb{Z} \rightarrow B \Gamma \rightarrow B G \rightarrow B H$, where the first map is the classifying map for the universal covering (that is, the identity if we use the model $B \mathbb{Z}=S^{1}$ ), and the other maps are induced by the inclusion $\mathbb{Z} \hookrightarrow \Gamma \hookrightarrow G \hookrightarrow H$ (the first inclusion sends $1 \in \mathbb{Z}$ to $1 \in \mathbb{Q} \subset \Gamma$ ).

Let $\bar{M} \rightarrow S^{1}$ be the induced covering, and $\bar{D}$ the lift of $D$ to this covering. Then, by the formula for delocalized eta invariants of induced manifolds,

$$
\eta_{<1>}(\bar{D})=\eta_{P}(\tilde{D})
$$

with $P=\mathbb{N}_{>0} \subset \mathbb{Z}$, which is not convergent by the above calculation.

This is an example of an operator where the delocalized eta invariant of John Lott is not defined.

Remark 3.1. The same calculation works for the product with product metric of a manifold $M$ of dimension $4 k$ with $S^{1}$. During the calculations, one has to multiply the above expressions for $S^{1}$ with $\hat{A}(M)$. If this number is nonzero, one therefore gets the same nonconvergence behavior for manifolds of arbitrarily high dimension.

Similar calculations should also be possible for more general mapping tori of a $4 k$-manifold, compare [Lott 1999]. One should be able to work with the signature as well as the Dirac operator.

Remark 3.2. It is probably not trivial to obtain an example where the conjugacyclass (inside the new group $\Gamma$ ) has polynomial growth. Observe that this is not the case for the construction we describe.

It would also be very interesting to find examples of nonconvergence with positive scalar curvature (then, necessarily, the conjugacy class could not have polynomial growth). 
It would be even more interesting if one could produce examples as above where the fundamental group of the manifold is the group $H$. It is not clear to us how to construct such an example and keep control of the calculation of the $\eta$-invariant.

Another open problem is the construction of examples with nonconvergent delocalized $L^{2}$-Betti numbers. As a starting point, one should again look for manifolds with many nontrivial such numbers; by induction to larger groups one might then be able to obtain one conjugacy class where the invariants don't converge.

\section{Possible values of APS-rho invariants for the Dirac operator}

In this section, we prove Theorem 1.8. Its proof is modeled on the proof of the corresponding statement [Guentner et al. 2005, Theorem 7.1] for the signature operator.

Let $M$ be a closed spin manifold with positive scalar curvature. Let $u: M \rightarrow$ $B \Gamma$ be a continuous map and $\lambda_{1}, \lambda_{2}: \Gamma \rightarrow U(d)$ two finite-dimensional unitary representations of $\Gamma$. Set $\Gamma_{1}:=\operatorname{im}\left(\lambda_{1}\right)$ and $\Gamma_{2}:=\operatorname{im}\left(\lambda_{2}\right)$. We consider $\Gamma_{1}$ and $\Gamma_{2}$ as discrete groups which happen to be subgroups of $U(d)$.

We compose $u$ with the maps induced by $\lambda_{1}$ and $\lambda_{2}$ to get $v: M \rightarrow B\left[\Gamma_{1} \times \Gamma_{2}\right]$. The tuple $(M, v)$ then represents an element $[M, v]$ in the real K-homology of $B\left[\Gamma_{1} \times \Gamma_{2}\right]$. We can now apply the reduced Baum-Connes map $\mu_{\text {red }}$ to this element to get

$$
\operatorname{ind}\left(D_{L}\right) \in K O_{*}\left(C_{\mathrm{red}}^{*}\left(\Gamma_{1} \times \Gamma_{2}\right)\right) .
$$

Here $L$ is the Mishchenko-Fomenko line bundle associated to $v$.

Since $M$ has positive scalar curvature, this index is zero by the Lichnerowicz formula. On the other hand, $\Gamma_{1} \times \Gamma_{2}$ is a linear group by its very construction. By the main result (0.1) of [Guentner et al. 2005], the following map

$$
\mu_{\mathrm{red}}: K_{*}^{\Gamma_{1} \times \Gamma_{2}}\left(E\left[\Gamma_{1} \times \Gamma_{2}\right]\right) \rightarrow K_{*}\left(C_{\mathrm{red}}^{*}\left(\Gamma_{1} \times \Gamma_{2}\right)\right)
$$

is split injective in this case. Their proof applies in the same way to the real Ktheory, since they really prove that linear groups uniformly embed into Hilbert space, which implies the coarse Baum-Connes isomorphism conjecture for linear groups. This in turn implies the real coarse Baum-Connes conjecture for linear groups by a well-known principle, compare for example [Schick 2004]. From here, the descent principle implies split injectivity of the usual real reduced BaumConnes map. Therefore

$$
K O_{*}^{\Gamma_{1} \times \Gamma_{2}}\left(E\left[\Gamma_{1} \times \Gamma_{2}\right]\right) \rightarrow K O_{*}\left(C_{\mathbb{R}, \text { red }}^{*}\left(\Gamma_{1} \times \Gamma_{2}\right)\right)
$$

is also injective. 
We must produce a link between $K O_{*}^{\Gamma_{1} \times \Gamma_{2}}\left(E\left[\Gamma_{1} \times \Gamma_{2}\right]\right)$ and the nonequivariant Baum-Connes map with $K O_{*}\left(B\left[\Gamma_{1} \times \Gamma_{2}\right]\right)$ used so far. There is a canonical map

$$
K O_{*}\left(B\left[\Gamma_{1} \times \Gamma_{2}\right]\right) \rightarrow K O_{*}^{\Gamma_{1} \times \Gamma_{2}}\left(E\left[\Gamma_{1} \times \Gamma_{2}\right]\right) .
$$

By standard arguments (compare [Guentner et al. 2005, Section 7] and [Lück and Stamm 2000, Lemma 2.9]) in equivariant homology theory, this map is split injective after tensoring with $R$ of Theorem 1.8.

Putting these two facts together,

$$
\mu_{\text {red }}: K O_{*}\left(B\left[\Gamma_{1} \times \Gamma_{2}\right]\right) \otimes R \rightarrow K O^{*}\left(C_{\text {red }}^{*}\left(\Gamma_{1} \times \Gamma_{2}\right)\right) \otimes R
$$

is injective. Since we have already seen that $\operatorname{ind}\left(D_{L}\right)=0$, this implies that there is $l \in \mathbb{N}$ that is a product of orders of elements of $\Gamma_{1} \times \Gamma_{2}$ such that

$$
l \cdot[M, u]=0 \in K O_{*}\left(B\left[\Gamma_{1} \times \Gamma_{2}\right]\right) .
$$

We now use the geometric description of $K O_{*}(X)$ in terms of spin bordism due to Hopkins and Hovey [1992, Theorem 1]. First observe that there is a natural map $\Omega_{*}^{\text {spin }}(X) \rightarrow K O_{*}(X)$ which assigns to a spin manifold $M$ with map $v: M \rightarrow X$ the class $[M, v] \in K O_{*}(X)$ given by the geometric description of $K O_{*}(X)$. Next, consider the special case $\tau: \Omega_{*}^{\text {spin }}(\mathrm{pt}) \rightarrow K O_{*}(\mathrm{pt})$ of this homomorphism for $X$ equal to a point. This is a (graded) ring homomorphism with kernel consisting of some manifolds with vanishing $\hat{A}$-genus, and cokernel the ideal generated by $K O_{-8}(\mathrm{pt})$. We can consider $K O_{*}(\mathrm{pt})$ as a module over $\Omega_{*}^{\text {spin }}(\mathrm{pt})$ via $\tau$ and form $\Omega_{*}^{\text {spin }}(X) \otimes_{\Omega_{*}^{\text {spin }}(\mathrm{pt})} K O_{*}(\mathrm{pt})$. The result of Hopkins and Hovey says that for each $\mathrm{CW}$-complex $X$ the induced map is an isomorphism:

$$
\Omega_{*}^{\mathrm{spin}}(X) \otimes_{\Omega_{*}^{\text {spin }}(\mathrm{pt})} K O_{*}(\mathrm{pt}) \stackrel{\cong}{\rightrightarrows} K O_{*}(X) .
$$

Definition 4.1. A Bott manifold $B$ is an eight-dimensional simply connected spin manifold with $\hat{A}(B)=1$.

Lemma 4.2. If $[M, v]=0 \in K O_{*}(X)$ then there are $n \in \mathbb{N}$ and spin manifolds $A_{i}, C_{i}$ with $\hat{A}\left(C_{i}\right)=0$ and continuous maps $u_{i}: A_{i} \rightarrow X$ such that $[M, v] \times B^{n}$ is bordant in $\Omega_{*}^{\text {spin }}(X)$ to the disjoint union of $\left[A_{i} \times C_{i}, u_{i}\right]$. The maps to $X$ are given by first projecting to the first factor and then using $v$ or $u_{i}$, respectively.

Proof. Considering $K O_{*}(\mathrm{pt})$ as a module over $\Omega_{*}^{\mathrm{spin}}(\mathrm{pt})$ as above, we obtain a split exact sequence of graded $\Omega_{*}^{\text {spin }}(\mathrm{pt})$-modules

$$
0 \rightarrow D \rightarrow \Omega_{*}^{\text {spin }}(\mathrm{pt})[x] /(B x-1) \rightarrow K O_{*}(\mathrm{pt}) \rightarrow 0,
$$

where the middle term is the quotient of the polynomial ring by the ideal generated by $(B x-1)$, where $B$ is the Bott manifold and where $x$ has degree -8 and is mapped to the generator of $K O_{-8}(\mathrm{pt})$. Note that $\Omega_{*}^{\text {spin }}(\mathrm{pt})[x] /(B x-1)$ is actually 
the localization $\Omega_{*}^{\text {spin }}(\mathrm{pt})\left[B^{-1}\right]$, where we invert $B$. The split is determined by the inverse of $\tau$ in degrees 0 through 7 (where $\tau$ is invertible), and by mapping the generator of $\mathrm{KO}_{8}(\mathrm{pt})$ to $B$.

Every element in $\Omega_{*}^{\text {spin }}(\mathrm{pt})\left[B^{-1}\right]$ can be written (nonuniquely) as $x^{k}[M]$ for a suitable spin manifold $M$ (because of the simple form of the relation, one can multiply every monomial with $B^{j} x^{j}$ to make any polynomial homogeneous and represent the same element in the quotient). Because the image of $x$ in $K O_{*}(\mathrm{pt})$ is a unit, the kernel $D$ consists therefore of elements of the form $[M] x^{k}$ with $\hat{A}(M)=0$.

Tensor now (4.3) with $\Omega_{*}^{\text {spin }}(X)$ to obtain a short split exact sequence of abelian groups:

$$
\begin{aligned}
0 \rightarrow \Omega_{*}^{\text {spin }}(X) \otimes_{\Omega_{*}^{\text {spin }}(\mathrm{pt})} D & \\
\rightarrow & \Omega_{*}^{\text {spin }}(X)\left[B^{-1}\right] \rightarrow \Omega_{*}^{\text {spin }}(X) \otimes_{\Omega_{*}^{\text {spin }}(\mathrm{pt})} K O_{*}(\mathrm{pt}) \rightarrow 0 .
\end{aligned}
$$

Observe now that, by the universal property of the localization, the $\Omega_{*}^{\text {spin }}(\mathrm{pt})$ module homomorphism $\Omega_{*}^{\text {spin }}(X) \rightarrow \Omega_{*}(X) \otimes_{\Omega_{*}^{\text {spin }}(\mathrm{pt})} K O_{*}(\mathrm{pt}) \cong K O_{*}(X)$ factors through $\Omega_{*}^{\text {spin }}(X)\left[B^{-1}\right]$ because $B$ is mapped to an invertible element of $K O_{*}(\mathrm{pt})$. Every element in the kernel of the projection $\Omega_{*}^{\text {spin }}(X)\left[B^{-1}\right] \rightarrow K O_{*}(X)$ is a product of $B^{-k}$ with a disjoint union of elements $\left[A_{i} \times C_{i}, u_{i}\right]$ as in the statement of the lemma. By assumption, $[M, v]$ is mapped to such an element in the localization $\Omega_{*}^{\text {spin }}(X)\left[B^{-1}\right]$. Finally, two elements in such a localization are equal if they are bordant (that is, equal in $\Omega_{*}^{\text {spin }}(X)$ ) after multiplication with a sufficiently high power of $B$. This finishes the proof of the lemma.

We conclude that, since $l \cdot[M, v]=0 \in K O_{*}\left(B\left[\Gamma_{1} \times \Gamma_{2}\right]\right)$, there are product manifolds $A_{i} \times C_{i}$ with $\hat{A}\left(C_{i}\right)=0$ and with continuous maps $u_{i}: A_{i} \rightarrow B\left[\Gamma_{1} \times \Gamma_{2}\right]$ and $n \in \mathbb{N}$ such that $l \cdot[M, v] \times B^{n}$ is bordant in $\Omega_{*}^{\text {spin }}\left(B\left[\Gamma_{1} \times \Gamma_{2}\right]\right)$ to $\sum\left[A_{i}, u_{i}\right] \times C_{i}$. Let $[W, f]$ be the corresponding bordism. Note that the flat bundles associated to $\lambda_{1}$ and $\lambda_{2}$ pull back from $M$ to each copy of $M \times B^{n}$ in $\partial W$ and extend to all of $W$, restricting on $A_{i} \times C_{i}$ to flat bundles that pull back from $A_{i}$.

By the multiplicativity of eta invariants (compare [Gilkey 1984]),

$$
\rho_{\lambda_{1}-\lambda_{2}}\left(M \times B^{n}\right)=\rho_{\lambda_{1}-\lambda_{2}}(M) \cdot \hat{A}\left(B^{n}\right)=\rho_{\lambda_{1}-\lambda_{2}}(M) .
$$

On the other hand,

$$
\rho_{\lambda_{1}-\lambda_{2}}\left(A_{i} \times C_{i}\right)=\rho_{\lambda_{1}-\lambda_{2}}\left(A_{i}\right) \hat{A}\left(C_{i}\right)=0 .
$$

By the classical Atiyah-Patodi-Singer index theorem, the difference of the APSindices of the Dirac operator on $W$ twisted with the flat bundles associated to $\lambda_{1}$ and $\lambda_{2}$ is an integer, which is equal to the difference of the rho invariants of $l \cdot M \times B^{n}$ 
and of $A_{i} \times C_{i}$ (indeed, the local terms will cancel out). To conclude,

$$
l \cdot \rho_{\lambda_{1}-\lambda_{2}}(M) \in \mathbb{Z}
$$

and this is exactly what we had to prove.

We remark that the calculation of $K O_{*}(X)$ in terms of spin-bordism of [Hopkins and Hovey 1992] is very nontrivial, and is crucially used in our argument.

\section{Acknowledgements}

Part of this work was carried out during visits of the authors to Göttingen and Rome funded by Ministero Istruzione Università Ricerca, Italy (Cofin Spazi di Moduli e Teorie di Lie) and DFG-Graduiertenkolleg "Gruppen und Geometrie" (Göttingen).

\section{References}

[Botvinnik and Gilkey 1995] B. Botvinnik and P. B. Gilkey, "The eta invariant and metrics of positive scalar curvature”, Math. Ann. 302:3 (1995), 507-517. MR 96f:58159 Zbl 0835.58034

[Botvinnik and Gilkey 1996] B. Botvinnik and P. B. Gilkey, "Metrics of positive scalar curvature on spherical space forms", Canad. J. Math. 48:1 (1996), 64-80. MR 97c:58155 Zbl 0859.58026

[Chang and Weinberger 2003] S. Chang and S. Weinberger, "On Invariants of Hirzebruch and Cheeger-Gromov", Geom. Topol. 7 (2003), 311-319.

[Gilkey 1984] P. B. Gilkey, Invariance theory, the heat equation, and the Atiyah-Singer index theorem, Mathematics Lecture Series 11, Publish or Perish, Wilmington, DE, 1984. MR 86j:58144 Zbl 0565.58035

[Guentner et al. 2005] E. Guentner, N. Higson, and S. Weinberger, "The Novikov conjecture for linear groups”, Publ. Math. Inst. Hautes Études Sci. 101 (2005), 243-268. MR MR2217050 Zbl 1073.19003

[Hopkins and Hovey 1992] M. J. Hopkins and M. A. Hovey, "Spin cobordism determines real $K$ theory”, Math. Z. 210:2 (1992), 181-196. MR 93g:55007 Zbl 0770.55008

[Keswani 2000] N. Keswani, "Von Neumann eta-invariants and $C^{*}$-algebra $K$-theory", J. London Math. Soc. (2) 62:3 (2000), 771-783. MR 2001k:58043 Zbl 1021.58018

[Lawson and Michelsohn 1989] B. Lawson and M.-L. Michelsohn, Spin geometry, Princeton University Press, 1989.

[Leichtnam and Piazza 1997] E. Leichtnam and P. Piazza, "The $b$-pseudodifferential calculus on Galois coverings and a higher Atiyah-Patodi-Singer index theorem”, Mém. Soc. Math. Fr. (N.S.) 68 (1997), iv+121. MR 99d:58168

[Leichtnam and Piazza 2001] E. Leichtnam and P. Piazza, "On higher eta-invariants and metrics of positive scalar curvature”, K-Theory 24:4 (2001), 341-359. MR 2002k:58051 Zbl 1010.58019

[Lott 1999] J. Lott, "Delocalized $L^{2}$-invariants", J. Funct. Anal. 169(1999), 1-31. MR 2000k:58039 Zbl 0958.58027

[Lott 2004] J. Lott, "Erratum to: "Delocalized $L^{2}$-invariants" [J. Funct. Anal. 169 (1999), 1-31; MR1726745]”, J. Funct. Anal. 210:1 (2004), 258. MR 2005a:58052

[Lück and Stamm 2000] W. Lück and R. Stamm, "Computations of $K$ - and $L$-theory of cocompact planar groups”, K-Theory 21:3 (2000), 249-292. MR 2001k:19004 Zbl 0979.19003 
[Piazza and Schick 2007] P. Piazza and T. Schick, "Bordism, rho-invariants and the Baum-Connes conjecture", J. Noncommut. Geom. 1:1 (2007), 27-111. MR MR2294190

[Rotman 1995] J. J. Rotman, An introduction to the theory of groups, Fourth ed., Graduate Texts in Mathematics 148, Springer, New York, 1995. MR 95m:20001 Zbl 0810.20001

[Schick 2004] T. Schick, "Real versus complex $K$-theory using Kasparov's bivariant $K K$-theory", Algebr. Geom. Topol. 4 (2004), 333-346. MR 2005f:19007 Zbl 1050.19003

[Stolz 1995] S. Stolz, "Positive scalar curvature metrics - existence and classification questions", pp. 625-636 in Proceedings of the International Congress of Mathematicians, Vol. 1, 2 (Zürich, 1994), Birkhäuser, Basel, 1995. MR 98h:53063 Zbl 0848.57021

[Stolz 1999] S. Stolz, "Concordance classes of positive scalar curvature metrics", preprint, Notre Dame Univ., 1999.

[Wiethaup 2004] M. Wiethaup, "Wachstum von Konjugiertenklassen in diskreten Gruppen”, Diplomarbeit Georg-August-Universität Göttingen, 2004, Available at http://www.uni-math.gwdg.de/ schick/publ/Diplom_moritz_wiethaup.dvi.

Received June 14, 2006. Revised September 21, 2006.

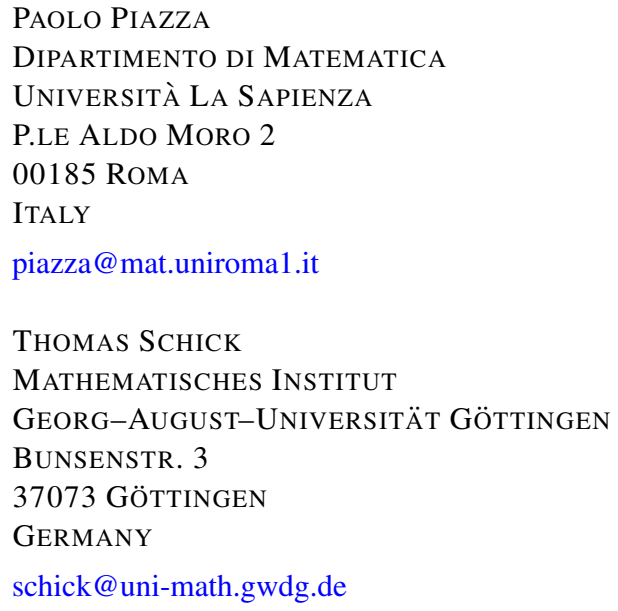

